\begin{abstract}
About Cigarettes
\section{Somchai Bovornkitti*}

Emeritus Professor of Medicine, Thailand

\section{Opinion}

A cigarette is a small cylinder of finely cut tobacco leaves rolled in thin paper, in tobacco or banana leaves. Another definition is a cylinder filled with cubeb or tobacco or marijuana. Cigarette could be classified into two categories, combustible cigarette and non-combustible cigarette or electronic cigarette. Combustion or burning is a differentiation. While combustible tobaccos release grayish white smoke, electronic cigarettes (cigarlike) produce vapor or aerosol mist.

Cigarette smoke from combustion of tobacco contains over 6,000 chemicals, among which about 100 are potentially harmful. Exposure to those chemicals (not nicotine) is the cause of smoking-related diseases. Without combustion, electronic cigarettes contain less toxicants and no tar. It is highly possible to use electronic cigarettes to assist the cessation of addiction to the burning tobacco cigarettes that emit of toxic substances including carcinogens that adversely impact smokers' health with their smoking related serious complications such as chronic bronchitis, pulmonary emphysema and bronchial cancers. To address the concerns of harm from tobacco smoke, there are several tobacco harm reduction strategies such as banish cigarette smoking, using the less harm e-cigarettes as an alternative, inventing cancer vaccine for smokers who are unable or unwilling to quit smoking or using advance technology such as DNA repair.
\end{abstract}

ISSN: 2689-2707

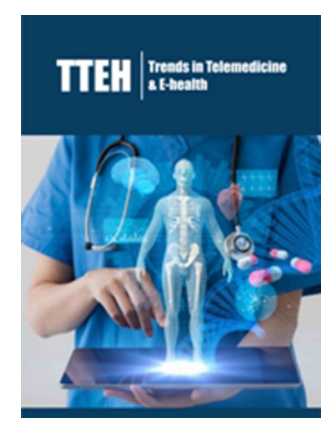

*Corresponding author: Somchai Bovornkitti, Emeritus Professor of Medicine, Fellow of the Academy of Science, The Royal Society of Thailand, Thailand

Submission: 監 August 26, 2019

Published: 制September 04, 2019

Volume 2 - Issue 1

How to cite this article: Somchai Bovornkitti. About Cigarettes. Trends Telemed E-Health 2(1). TTEH. 000527. 2019.

DOI: 10.31031/TTEH.2019.02.000527

Copyright@ Somchai Bovornkitti, This article is distributed under the terms of the Creative Commons Attribution 4.0 International License, which permits unrestricted use and redistribution provided that the original author and source are credited.

\section{End Note}

The Royal Society of Thailand is the highest academy among others in the country.

For possible submissions Click below: 